\title{
Primary Health Care Providers' Perspectives on Developing an eHealth Tool for Physical Activity Counselling: A Qualitative Study
}

This article was published in the following Dove Press journal: Journal of Multidisciplinary Healthcare

\author{
Apichai Wattanapisit (D) ${ }^{1,2}$ \\ Sanhapan Wattanapisit ${ }^{3}$ \\ Titiporn Tuangratananon ${ }^{4}$ \\ Waluka Amaek ${ }^{5}$ \\ Sunton Wongsiri ${ }^{6}$ \\ Prachyapan Petchuay'
}

'Department of Clinical Medicine, School of Medicine, Walailak University, Nakhon Si Thammarat, Thailand; ${ }^{2}$ Family Medicine Clinic, Walailak University Hospital, Nakhon Si Thammarat, Thailand; ${ }^{3}$ Primary Care Unit, Thasala Hospital, Nakhon Si Thammarat, Thailand; ${ }^{4}$ International Health Policy Program, Ministry of Public Health, Nonthaburi, Thailand; ${ }^{5}$ College of Graduate Studies, Walailak University, Nakhon Si Thammarat, Thailand; ${ }^{6}$ Department of Orthopedics, Faculty of Medicine, Prince of Songkla University, Songkhla, Thailand

Correspondence: Apichai Wattanapisit School of Medicine, Walailak University, 222, Thaiburi, Thasala, Nakhon Si

Thammarat, 80161 , Thailand

Tel +66 7567280 l

Fax +6675672807

Email apichai.wa@wu.ac.th
Purpose: Physical inactivity is a global health concern. Physical activity (PA) counselling is an effective intervention for promoting PA in primary health care (PHC) settings. The use of electronic health (eHealth) technology has the potential to support PA counselling. This study aimed to explore PHC providers' perspectives on the development of an eHealth tool to aid PA counselling in the resource-limited settings.

Methods: This qualitative study employed interpretive phenomenology. The study was conducted at hospital-based PHC clinics among physicians and registered nurses. Data collection involved in-depth interviews (IDIs) and focus group discussions (FGDs). An inductive thematic approach was used to analyze the data.

Results: Three physicians participated in three IDIs and 12 nurses participated in four FGDs at three hospitals. The median age of the participants was 43 years. Participants saw 15-100 patients/day (median 40) and spent 2-20 min with each patient (median 5). Three themes emerged. Theme 1: requirements for PA counselling: the participants reflected the needs and characteristics of eHealth tool that may support PA counselling. Theme 2: enabling an eHealth tool for PA counselling: the eHealth should be easy to use, provide PA prescription function, and support follow-up PA counselling. Theme 3: reducing barriers to PA counselling: the eHealth tool was expected to help reduce service and workforce barriers and patients' limitations.

Conclusion: A well-designed and practical eHealth tool has the potential to improve PA counselling practice in PHC settings. The eHealth tool may affect an indirect mechanism to reduce barriers to PA counselling. Future research should focus on the usability and utility as well as the process evaluation of the PA counselling eHealth tool that will be implemented in resource-limited settings.

Keywords: counselling, eHealth, exercise, physical activity, primary health care

\section{Introduction}

Primary health care (PHC) is a suitable setting for modifying patients' lifestyles and health behaviors. ${ }^{1}$ PHC settings offer a first contact between providers and a large number of population. ${ }^{2,3}$ In addition, PHC providers are perceived as a reliable and credible source of health information. ${ }^{4}$ Promoting physical activity (PA) in PHC is a potential approach for increasing participation in PA. Several PA promotion programs are implemented in PHC settings around the world (eg Exercise is Medicine $^{\circledR}$ in Australia, Canada, Poland, Singapore, USA; Green Prescription in New Zealand; Let's Get Moving in UK). ${ }^{5}$ Previous literature supports that PA 
counselling is an effective intervention for promoting PA in PHC settings. ${ }^{6-8}$ PA counselling should be tailored to PHC settings and service users. ${ }^{7,9}$ However, a number of barriers to PA counselling remain, including those related to health care providers (eg lack of knowledge and skills), patients (eg insufficient time and support and personal limitations), and systems (eg time constraints and limited resources). ${ }^{4,6,9,10}$

Several promising technologies have been introduced and utilized in PHC settings. ${ }^{11}$ Specifically, electronic health (eHealth), which is defined as "the use of information and communications technology in support of health and health-related fields", ${ }^{12}$ has been applied for various purposes in PHC settings. ${ }^{13}$ In addition, mobile health (mHealth) is a type of eHealth, which is defined as "the use of mobile wireless technologies for health." ${ }^{, 12}$ mHealth has the potential to be used to support numerous clinical tasks in PHC settings, especially health promotion tasks. ${ }^{14}$

Evidence indicates that adopting eHealth and mHealth tools for PA counselling contributes to positive outcomes for patients and health care providers in the context of PHC settings (eg easiness and usefulness of eHealth programs, high satisfaction among patients and health care providers, improvement of PA participation). ${ }^{15-22}$ On the other hand, some studies continue to debate the usability and utility of eHealth for PA counselling in PHC (eg technical errors, time consuming, no improvement of PA participation). ${ }^{23-25}$ This reflects the diverse requirements in different PHC settings.

In Thailand, Thai people can access to health care services under the universal health coverage. ${ }^{26}$ District hospitals serve outpatient and PHC clinics for the population in their catchment areas. Two major challenges of providing the hospital-based PHC clinics are a heavy workload and time constraints due to the limited number of staff. For example, a physician in district hospital sees about 56 patients per day and spends about $3.8 \mathrm{~min}$ per consultation. ${ }^{4}$ Moreover, within this specific setting, exercise specialists are not common health professional in district hospitals. A small number of physical therapists are available in district hospitals. The physical therapists take responsibility for disease-specific care rather than performing general PA counselling in PHC. Physicians and nurses are dominant providers for PA counselling in PHC. These are the challenges of implementing PA counselling in the resource-limited clinical settings.

To the best of our knowledge, eHealth tools for PA counselling have been developed and utilized in high- income countries. An appropriate eHealth tool may have the potential to support PA counselling in resource-limited clinical settings. Therefore, this raises a research question: what are the essential elements for developing an eHealth tool to support PA counselling in the resource-limited PHC settings? This study aimed to explore perspectives of $\mathrm{PCH}$ providers on the development of an eHealth tool to aid PA counselling in the resource-limited PHC settings.

\section{Methods \\ Study Design}

This qualitative study was part of an eHealth development project for PA counselling in PHC. The qualitative part was conducted between February and March 2020. The ontological assumptions aligned with relativism (ie reality is subjective and differs from person to person). ${ }^{27}$ The epistemological assumptions were subjectivism (ie knowledge is based on real-world phenomena). ${ }^{27}$ The qualitative approach was interpretive phenomenology, which aimed to the contextual features of an experience of the phenomenon. ${ }^{28}$ The Consolidated Criteria for Reporting Qualitative Research and the Standards for Reporting Qualitative Research were used as guidelines regarding transparently utilizing qualitative methodologies and writing the manuscript. ${ }^{29,30}$

\section{Context and Participants}

The study was conducted at hospital-based noncommunicable disease (NCD) clinics (primary care clinics for patients with hypertension or diabetes mellitus) at three district hospitals in Nakhon Si Thammarat province, Thailand. The three hospitals represented small $(\leq 30$ beds), medium (30-120 beds), and large ( $\geq 120$ beds) community hospitals, respectively. The community hospitals in the same province were selected to acquire the similar practice of NCD clinics in the different size of hospitals to achieve transferability of the study. The authors contacted the hospital directors and heads of NCD clinics to explain the project summary and requested permission to collect data from staff of all NCD clinics. All four NCD clinics were operated in small (one), medium (one), and large (two) community hospitals.

Physicians and nurses contacted patients during the care processes at the NCD clinics. Figure 1 illustrates a summary of the patient flow in the NCD clinics at the three hospitals. Both professionals had opportunities to counsel patients about NCD management, including PA. There was no 


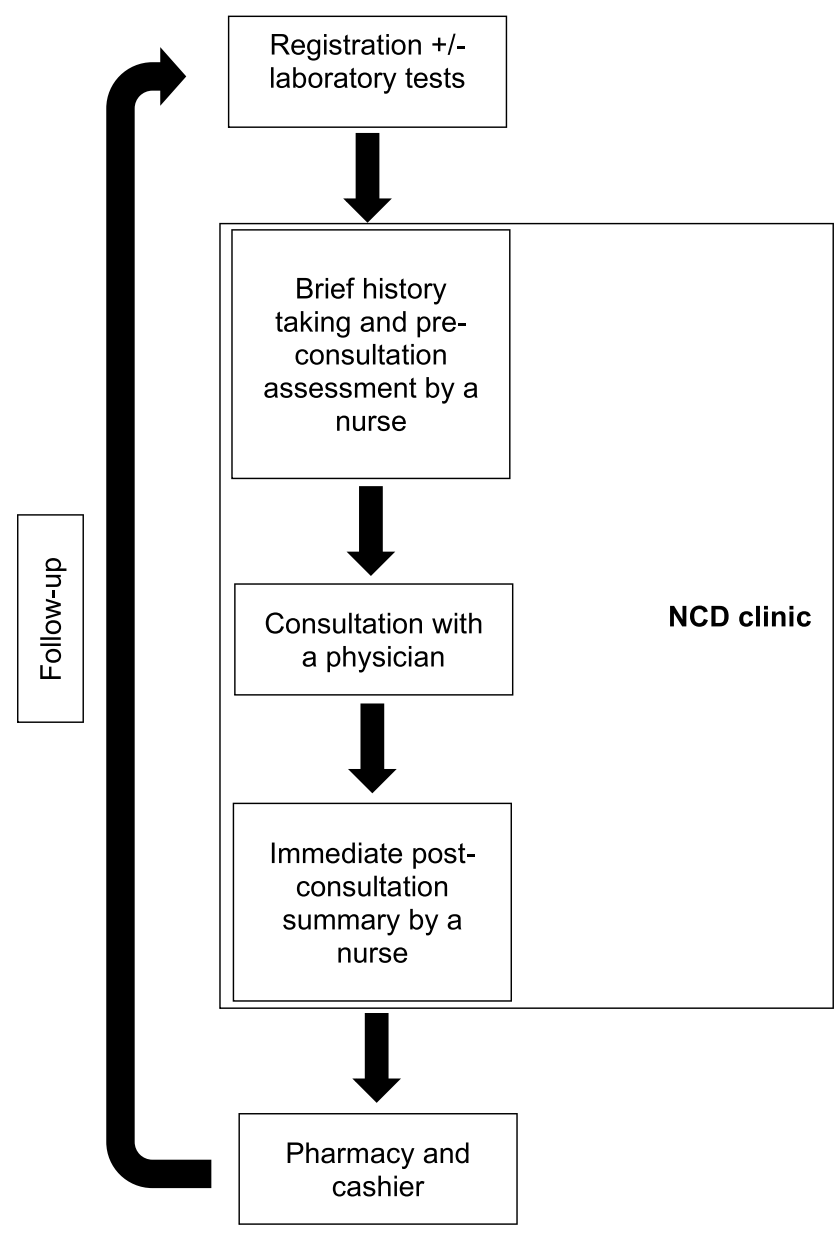

Figure I Patient flow in NCD clinics.

official PA counselling guideline for NCD clinics. Nurses may involve some parts of PA counselling, such as assessing patient's PA participation and providing brief advice on PA. Physicians may have a chance for an additional part of PA counselling (ie PA prescription). Using purposive sampling, male and female general practitioners (GPs; physicians who were not certified in any medical specialty) or family physicians (physicians who were certified as family medicine specialists) who operated the NCD clinics were invited to participate in in-depth interviews (IDIs). Registered nurses at the NCD clinics were also recruited to participate in focus group discussions (FGDs).

\section{Data Collection}

Both physicians and nurses were invited to participate in this qualitative study to obtain the data triangulation and credibility of the data. One of the authors (AW), a male family physician who has undergone training in and has experience in qualitative research, conducted all the IDIs and FGDs in Thai. A research assistant with training in audio transcription took notes. To achieve dependability, all IDIs and FGDs were conducted at the NCD clinics (the participants' worksites) at times that were convenient to the participants. The physicians took part in IDIs, while each FGD consisted of two to four nurses, depending on the participants' availability and preferences. Each IDI or FGD took 30-60 min and was recorded by two digital audio recorders with the verbal permission of the participants. In each hospital, the authors conducted the $\mathrm{FGD}(\mathrm{s})$ to obtain the data from nurses who were responsible for pre- and post-consultation (Figure 1). Thereafter, the authors interviewed a physician to explore the perspectives on the consultation.

The semi-structured interview guide, generated by the authors and reviewed by the Human Research Ethics Committee of Walailak University, was used to guide the IDIs and FGDs (Supplementary file). The number of IDIs and FGDs conducted was based on data saturation, as they were discontinued once new data reproduced what was already found based on previous data. ${ }^{31}$

\section{Data Analysis}

The audio-recorded files were transcribed verbatim (wordfor-word) by the notetaker and checked by the interviewer (AW). The transcripts were managed using Microsoft Word (Office 365 University package, Microsoft Inc., Redmond, WA, USA), and hard copies were printed. The participants' identifiers were coded to ensure anonymity and confidentiality. An inductive thematic approach was used to analyze the data. ${ }^{32,33}$ The following processes were described to ensure confirmability of the study. Two authors (AW and SW, a family physician with experience in qualitative research) read the transcripts independently to familiarize themselves with the data and generated initial codes for the text throughout the transcripts. Subsequently, the initial codes were combined to develop initial thematic maps. The two authors collaborated in defining and naming the final themes. Another two authors (TT, who is a GP, and WA, who is an industrial designer) were involved in resolving any disagreements regarding the data analysis. All authors verified the data analysis and a consensus was reached. AW translated the themes and relevant quotations from Thai to English at the time of manuscript writing. Finally, all authors approved the translation.

\section{Ethical Approval}

The study protocol was approved by the Human Research Ethics Committee of Walailak University (approval 
number: WUEC-19-227-01). Participant information sheets were provided, and participation in the study was voluntary. The participants signed written consent forms prior to participating in the study. The presentation of participants' information in the published article was anonymized. This study was conducted in accordance with the Declaration of Helsinki.

\section{Results}

Three IDIs (involving three physicians) and four FGDs (involving 12 nurses) were conducted at the three hospitals. Among the 15 participants (13 females and 2 males), the median age was 43 years. The median experience at NCD clinics was 2 years. The physicians and nurses saw a median of 46 patients per day and reported spending a median of 5 min with each patient. Most participants $(80 \%, n=12)$ had no experience of using technology for PA counselling. Three participants seldom advised patients to use existing technologies for promoting PA, such as video clips that demonstrated exercise programs and step counter applications on mobile phones. All participants had no experience of using an innovative tool designed for PA counselling in clinical settings. Table 1 presents the participant codes and characteristics.

The findings comprised three major themes: (i) requirements for PA counselling; (ii) enabling an eHealth tool for PA counselling; and (iii) reducing barriers to PA counselling. Table 2 presents the summary of the details of initial codes and final themes. The three themes were interlinked, influencing each other. Figure 2 illustrates the conceptual map of the findings.

\section{Theme I: Requirements for PA Counselling}

PA counselling was considered an essential element at the NCD clinics. The PHC providers considered PA to be a beneficial lifestyle modification for NCD management, especially for patients with uncontrolled conditions. However, official PA counselling guidelines were not established for NCD clinics. The PHC providers required a tool that supported their services.

\section{An Evidence-Based eHealth Tool}

The majority of the providers were not confident about giving advice on PA for patients with specific diseases, especially for elderly individuals with poorly controlled conditions. No clinical or practical guidance on PA counselling was offered to the providers at the NCD clinics. It was impossible for the providers to know everything about PA. The eHealth tool should aid PA counselling based on the evidence-based information.

I am able to counsel healthy people (about PA), but I am not sure how to deal with patients with other underlying diseases (other than diabetes mellitus and hypertension).

Table I Participant Codes and Characteristics $(n=15)$

\begin{tabular}{|c|c|c|c|c|c|c|c|c|}
\hline $\begin{array}{l}\text { Participant } \\
\text { Code }\end{array}$ & $\begin{array}{l}\text { Method } \\
\text { Code }\end{array}$ & $\begin{array}{l}\text { Hospital } \\
\text { Size }\end{array}$ & Gender & $\begin{array}{l}\text { Age } \\
\text { (Year) }\end{array}$ & $\begin{array}{l}\text { Experience at } \\
\text { NCD Clinics } \\
\text { (Year) }\end{array}$ & $\begin{array}{l}\text { Number of } \\
\text { Patients } \\
\text { (per Day) }\end{array}$ & $\begin{array}{l}\text { Time Spent } \\
\text { per Patient } \\
\text { (Minute) }\end{array}$ & $\begin{array}{l}\text { Experience of Using } \\
\text { Technology for PA } \\
\text { Counselling }\end{array}$ \\
\hline $\mathrm{NI}$ & FGDI & Small & $\mathrm{F}$ & 43 & 10 & 30 & 5 & No \\
\hline N2 & FGDI & Small & $\mathrm{F}$ & 50 & 1 & 20 & 4 & Yes \\
\hline N3 & FGDI & Small & $F$ & 30 & I & 70 & 5 & Yes \\
\hline N4 & FGD2 & Medium & $F$ & 44 & 9 & 40 & 3.5 & No \\
\hline N5 & FGD2 & Medium & $F$ & 39 & 1.5 & 40 & 5 & No \\
\hline N6 & FGD3 & Large & $F$ & 48 & 5 & 40 & 5 & No \\
\hline N7 & FGD3 & Large & $F$ & 53 & 15 & 60 & 4 & No \\
\hline N8 & FGD3 & Large & $\mathrm{F}$ & 25 & 1 & 100 & 3 & No \\
\hline N9 & FGD4 & Large & $\mathrm{F}$ & 55 & 33 & 20 & 10 & No \\
\hline NIO & FGD4 & Large & $\mathrm{F}$ & 55 & 6 months & 35 & 5 & No \\
\hline NII & FGD4 & Large & $F$ & 43 & I month & 30 & 7.5 & No \\
\hline$N 12$ & FGD4 & Large & $F$ & 30 & 2 & 15 & 20 & No \\
\hline PI & IDII & Small & $M$ & 44 & 20 & 70 & 4 & No \\
\hline P2 & IDI2 & Medium & $M$ & 28 & 3 & 60 & 2 & Yes \\
\hline P3 & IDI3 & Large & $\mathrm{F}$ & 26 & 2 & 60 & 5 & No \\
\hline $\begin{array}{l}\text { Median } \\
\text { (Range) }\end{array}$ & & & & $\begin{array}{l}43 \\
(25-55)\end{array}$ & $2(0.08-33)$ & $40(15-100)$ & $5(2-20)$ & \\
\hline
\end{tabular}

Abbreviations: F, female; FGD, focus group discussion; IDI, in-depth interview; M, male; N, nurse; NCD, non-communicable disease; P, physician; PA, physical activity. 
Table 2 Summary of Codes and Themes

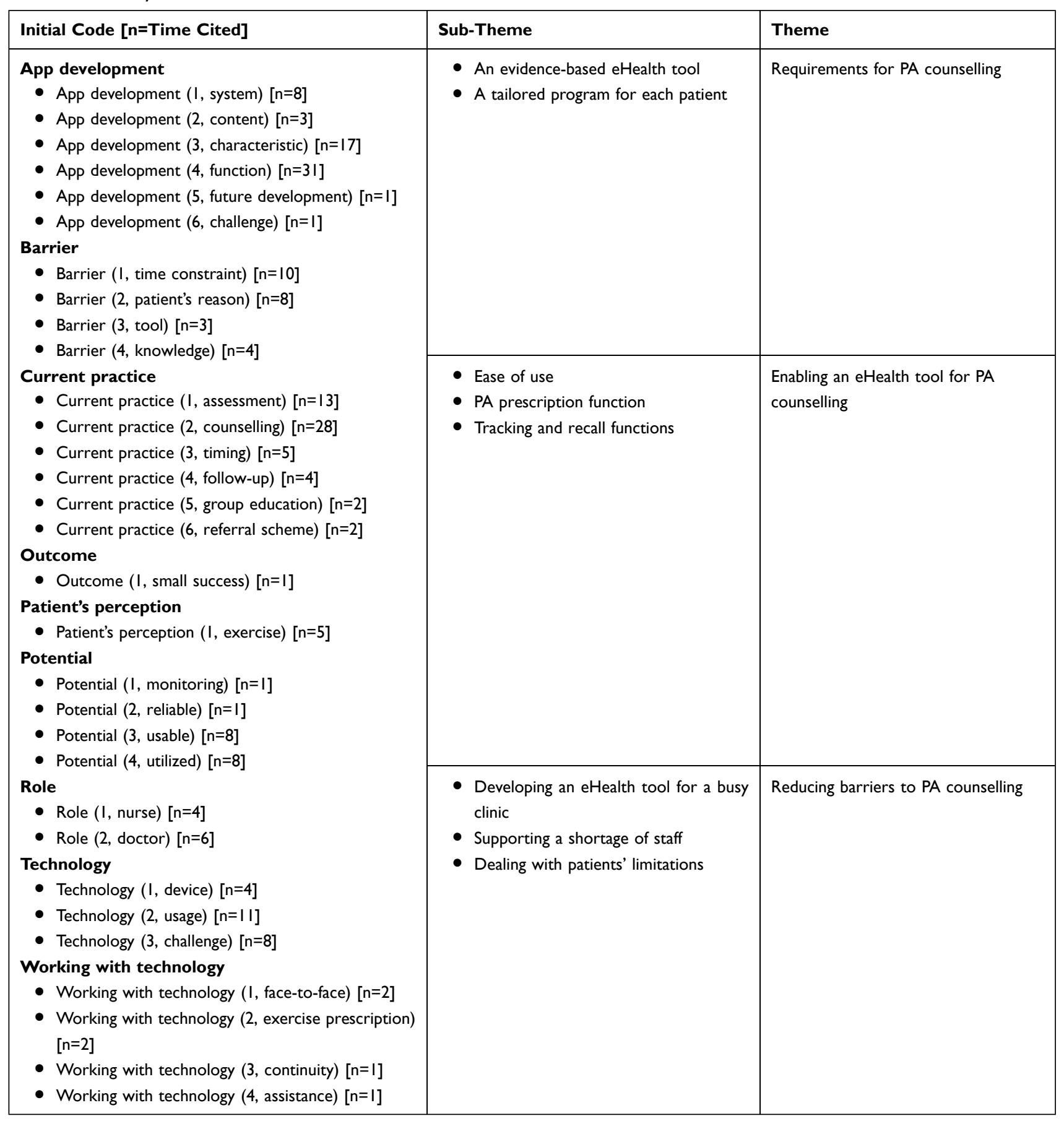

(N5, 1.5 years of experience, 40 patients/day, $5 \mathrm{~min} /$ patient)

We have no guidelines to design an exercise program for an individual. Moreover, we do not have the criteria to refer patients to a physiotherapist. Finally, I skip talking about exercise.

(N11, 1 month of experience, 30 patients/day, $7.5 \mathrm{~min} /$ patient)
If it (the eHealth tool) is accurate, it will be okay. I think it is good to try a new thing. If it works well, it will be great.

(N7, 15 years of experience, 60 patients/day, $4 \mathrm{~min} /$ patient)

\section{A Tailored Program for Each Patient}

A general recommendation on PA was suitable for general populations and healthy people. PA counselling for patients 


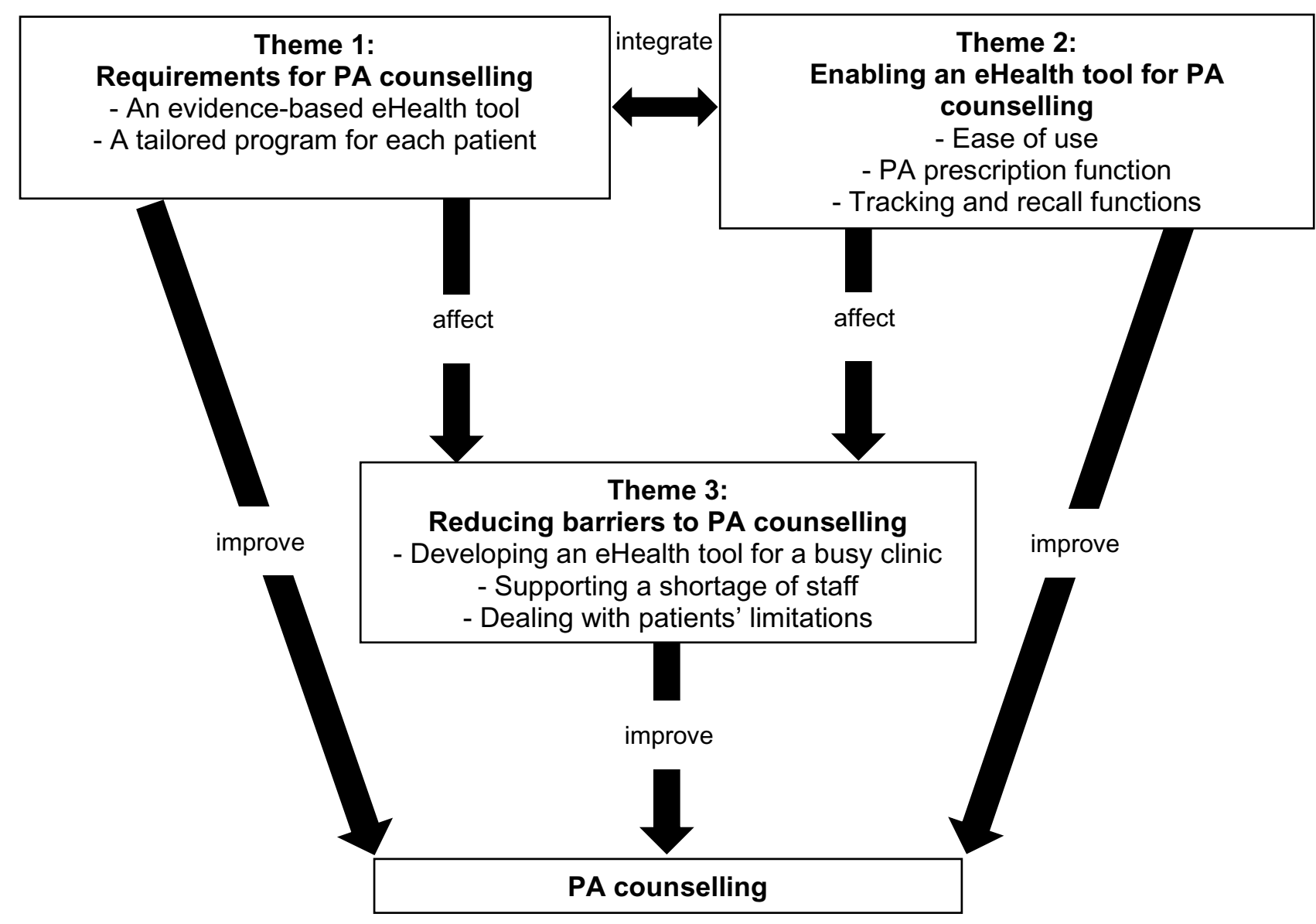

Figure 2 Summary of themes and conceptual map.

with NCDs was complex and specific for each person. Patients were different with different conditions. An eHealth tool would enable to perform PA counselling more efficiently.

The tool should provide a package of recommended activities for the patient, for example, the duration of walking or jogging. How can the patient measure and calculate the heart rate (intensity of an activity)? The tool may provide some examples, such as, "You feel tired and cannot speak words continuously". In other words, translating medical languages into plain language.

(P1, 20 years of experience, 70 patients/day, $4 \mathrm{~min} /$ patient)

The providers expected to see an eHealth tool that assisted PA counselling for patients with uncontrolled conditions (such as uncontrolled plasma glucose level, uncontrolled blood pressure, overweight and obesity). Patients in NCD clinics had some health problems. PA counselling was an additional therapy to combine with medications.
For a patient who has $150(\mathrm{mg} / \mathrm{dL})$ of plasma glucose, I will start talking about exercise and encourage the patient to exercise regularly. I ask the patient whether she is active. Some people are somewhat active, I will tell them to continue 30 min of walking for three to five days a week — a brisk walk -and evaluate the abilities of the patient, also her age. If the patient is totally inactive, I will recommend starting (exercise) slowly and gradually increasing the volume.

(N5, 1.5 years of experience, 40 patients/day, $5 \mathrm{~min} /$ patient)

If the plasma glucose is high, I will explore the causes. How is your diet control? If the diet and medication compliance are good, but there is no exercise, I will motivate the patient.

(N6, 5 years of experience, 40 patients/day, $5 \mathrm{~min} /$ patient)

I choose (to talk about PA with) patients who have comorbidities such as a BMI greater than $30\left(\mathrm{~kg} / \mathrm{m}^{2}\right)$ or high serum lipid or glucose.

(P2, 3 years of experience, 60 patients/day, $2 \mathrm{~min} /$ patient) 


\section{Theme 2: Enabling an eHealth Tool for PA Counselling}

Developing an eHealth tool for PA counselling requires understanding the clinical practice and service characteristics. The eHealth tool was expected to serve busy clinics as well as overcoming the challenges associated with implementing a new tool in clinical practice. Some participants were familiar with PA trackers or wearable devices. They used to advise patients to try those technologies for increase PA participation. Nevertheless, most participants could not foresee a new eHealth tool for PA counselling in NCD clinics.

\section{Ease of Use}

The providers expected an uncomplicated, concise, and non-time-consuming tool. The eHealth tool could be a patient-administered tool for assessing PA and its related factors or a provider-led assessment tool. Designing the tool for personal mobile devices may increase accessibility. An individual patient with their caregiver could utilize the tool in a waiting room.

The information is not much, not too many buttons, and easy to understand. It is a concrete idea, and patients can use it. It should not consume too much time. I can fill in some information, but if I must fill a full page, I will not.

(P2, 3 years of experience, 60 patients/day, $2 \mathrm{~min} /$ patient)

The best way is patients. Patients and their relatives (caregivers) complete the (eHealth) program and show the doctor the results. It is perfect because 1) it can save time, and 2) there is nothing to do in the waiting room - they can use the program. If the program is easy, it will be great. I think, finally, "self-care" or "self-management" is the answer.

(P1, 20 years of experience, 70 patients/day, $4 \mathrm{~min} /$ patient)

If the (eHealth PA counselling) program were on my computer screen, I could complete the information during the history taking.

(N4, 9 years of experience, 40 patients/day, $3.5 \mathrm{~min} /$ patient)

\section{PA Prescription Function}

The providers did not have a clear picture of a new eHealth tool for PA counselling. The concept of PA prescription was novel for the NCD clinics. PA prescription could be part of PA counselling. The generation of electronic PA prescriptions was highlighted as a promising function that could support PA counselling at the NCD clinics. Thus, the eHealth tool should assess the patient profile and generate a tailored PA prescription. In addition, the providers suggested providing multiple activity choices for each patient. The prescription could be a printout or a digital file.

I think the simplest way is a prescription, like a drug prescription. Then, the patient can manage himself/herself. It is very easy.

(P1, 20 years of experience, 70 patients/day, $4 \mathrm{~min} /$ patient)

Perhaps it should provide at least two to three types of activities.

(N5, 1.5 years of experience, 40 patients/day, $5 \mathrm{~min} /$ patient)

Only speech cannot be memorized. A printout or a written material can remind the patient. It can be either a printout or a digital file on a (mobile) app.

(P3, 2 years of experience, 60 patients/day, $5 \mathrm{~min} /$ patient)

\section{Tracking and Recall Functions}

The eHealth tool should support follow-up PA counselling. Some providers anticipated that the system would involve a built-in PA tracker on a mobile or wearable device, while others did not expect a tracking function to be available in the initial phase of the development of the tool. The providers mentioned the need for a recall function that would allow them to view previous results to inform follow-up counselling.

I expect to see a personal recommendation on a smartphone. A patient wears a pair of earphones and follows the recommendation (provided by the PA counselling eHealth tool).

(N2, 1 years of experience, 20 patients/day, $4 \mathrm{~min} /$ patient)

The (PA) assessment has been made this visit. We can see it at the next visit.

(N5, 1.5 years of experience, 40 patients/day, $5 \mathrm{~min} /$ patient)

\section{Theme 3: Reducing Barriers to PA Counselling}

There were some barriers to PA counselling in the resource-limited settings. An eHealth tool should be designed to deal with the barriers. Both patients and providers could get benefits from the tool. 


\section{Developing an eHealth Tool for a Busy Clinic}

An eHealth tool would be a supportive instrument for PA counselling. Nonetheless, using the tool may increase provider workload and disrupt routine services. The providers anticipated an eHealth tool that would be feasible for the resource-limited settings. Patients could learn to use a new technology.

It will increase our workload? We need to see whether it takes a long time? Initially, we have to advise patients (to use the tool). It will increase the workload, but it may be worth it in the long term.

(N5, 1.5 years of experience, 40 patients/day, $5 \mathrm{~min} /$ patient)

Patients follow the modern trend. They know how to chat with their families. They are able to search for information (on the internet). Some patients ask me about drug interactions. They learn to use technology more than they did in the past.

(N3, 1 year of experience, 70 patients/day, $5 \mathrm{~min} /$ patient)

The physicians and nurses saw many patients each day. This led to a short period of time to contact a patient at each point. A session for PA counselling would be very brief. It was a challenge and consideration for developing an eHealth tool for PA counselling.

Time is limited. If the doctor comes late, it is my "golden period" to mention exercise for 5 to $10 \mathrm{~min}$.

(N1, 10 years of experience, 30 patients/day, $5 \mathrm{~min} /$ patient)

Not more than one minute, is that enough? One and a half minutes, or one to two minutes (laughing).

(N5, 1.5 years of experience, 40 patients/day, $5 \mathrm{~min} /$ patient)

Well! The problem is the time. If the assessment takes only one more minute, it is okay. Taking 5-10 $\mathrm{min}$ is impractical because I do not have enough time. I wish to see a tool that can create information, and I will talk about some details. Then, the patient can go over (the information) at home.

(P2, 3 years of experience, 60 patients/day, $2 \mathrm{~min} /$ patient)

\section{Supporting a Shortage of Staff}

A limited number of staff was a major barrier to PA counselling. Two meanings were reflected. First, there were no exercise specialists in all hospitals. Physical therapists were not available for PA counselling at NCD clinics. A referral scheme for PA counselling among patients with NCDs was not common due to a shortage of staff. PA counselling could be a challenging task for NCD clinic providers. Second, the number of NCD clinic providers was not enough. Therefore, PA counselling was not considered as a first-line treatment for patients with NCDs. An eHealth tool should enable the NCD clinic providers to utilize the tool without the extra support of other staff.

I am not really confident about the in-depth physical activity. I know the basic concepts, but it is not comparable to occupational therapists or physiotherapists. Each patient has different limitations.

(N12, 2 years of experience, 15 patients/day, 2 $0 \mathrm{~min} /$ patient)

We cannot complete everything because of time limitation. Patients come to the hospital at 6.30 AM for blood tests, then history taking. We have only four staff. We have to record the information on the computer program. It is our routine. Most importantly, we cannot extend the time.

(N9, 33 years of experience, 20 patients/day, $10 \mathrm{~min} /$ patient)

\section{Dealing with Patients' Limitations}

Patient-related barriers to effective PA counselling were mentioned, including inaccurate perceptions regarding health conditions, cognitive issues, personal limitations, and lack of digital technology use. An eHealth tool should cover other aspects of changing health behaviors. For example, the tool may raise awareness of patients about their health conditions. Moreover, the eHealth tool should be usable for different groups of patients (eg age, gender). For a self-administered eHealth tool, it should not require a high-cost device. Otherwise, a provider-led tool was more appropriate for patients who could not afford personal devices.

The awareness of health is different among people. Some people who have a BMI of $30\left(\mathrm{~kg} / \mathrm{m}^{2}\right)$, they think they are not obese. They perceive like this. While some people with a BMI of $26\left(\mathrm{~kg} / \mathrm{m}^{2}\right)$, they have concern about obesity. For people who are aware of their fatness, I feel I can easily talk because they intend to change. On the other hand, people with a continuously high glucose level, poorly controlled, increased weight, and a lack of concern about diabetes, cannot change themselves. They are unaware. 
(P2, 3 years of experience, 60 patients/day, $2 \mathrm{~min} /$ patient)

Age and the background of the patient are important. I feel comfortable talking with the working-age population. For the elderly, it is challenging to communicate with them. Sometimes, they cannot hear clearly. Sometimes, I accidentally use medical terms. They do not understand.

(N12, 2 years of experience, 15 patients/day, $20 \mathrm{~min} /$ patient)

I think it (using a self-administered eHealth tool) is impossible for some patients. If you ask about the readiness, I think of two groups. The low-socioeconomic group is not ready. The high-income and educated group, of course, they can use it. However, older people are not interested in doing this.

(P2, 3 years of experience, 60 patients/day, $2 \mathrm{~min} /$ patient)

\section{Discussion \\ Principal Results}

This qualitative study presented the perspectives on developing an eHealth tool for PA counselling among health care providers at NCD clinics in the resourcelimited PHC settings. PA counselling was not a common practice due to a shortage of staff and heavy workload. The providers could not foresee a new eHealth tool for PA counselling. The findings of the study were interpreted into three major themes. First, there were needs to be supported to improve PA counselling in NCD clinics. An eHealth tool had the potential and would require some characteristics (ie evidence-based information, tailored program) to enhance the quality of PA counselling. Second, the PHC physicians and nurses expected to see an easy tool for patients and providers. The provision of individually tailored PA prescriptions, with multiple activity options, was expected. A recall function that supported follow-up counselling was suggested. Third, the eHealth tool should be developed to reduce barriers to PA counselling. The eHealth tool that would be able to support the busy clinics and a shortage of staff was recommended. In addition, the eHealth tool should be able to overcome a variety of limitations among patients.

\section{Comparison with Prior Work}

An early study explored the use of personal computers combined with the internet for assisting PA counselling in PHC. ${ }^{15}$ Thereafter, several types of eHealth technologies were used, for example, telephone-linked communication ${ }^{17}$ and smartphone applications. ${ }^{19-21,34-39}$ Numerous studies have explored PA as a stand-alone topic for counselling, ${ }^{17,19-21,34,37,39-42}$ while others have explored counselling for combinations of PA and other health behaviors (eg nutrition, smoking, and alcohol use). ${ }^{15,16,18,23,35,36,38,43-51}$ Among these studies, the outcomes of using eHealth technologies for PA counselling in PHC settings were diverse. ${ }^{52}$ This is reflective of the different needs across the wide range of PHC settings. Accordingly, the development of a new eHealth tool requires understanding providers' perspectives on current practice, required characteristics of the tool, and challenges.

Other than ensuring usability and utility of eHealth technologies, developing an eHealth tool for clinical purposes should involve considering issues around safety, quality, privacy, access, research, education, and ethics. ${ }^{53-55}$ The acceptance of a new technology in clinical settings depends on individuals' perceptions, knowledge, and experience. ${ }^{56,57}$ Factors that have been identified as being key for the successful implementation of any eHealth intervention in clinical settings include patient empowerment, self-management, and health care quality. ${ }^{58}$ However, using eHealth technologies may increase the workload of providers and disrupt the workflow of services. ${ }^{58}$ The issues reported in previous studies should be considered by eHealth developers.

The lessons learned from previous literature were valuable and applicable to design a new eHealth tool for PA counselling. However, most previous studies were conducted in high-income or developed countries. This qualitative study was conducted in a developing country to explore context-specific perspectives. Some findings (eg a short period of consultation, a lack of supportive staff and specialists) reflected the perspectives on the special concerns of developing an eHealth tool and needs of the study sites (four NCD clinics). The findings of the study could shed light on the understanding and consideration for developing eHealth tools for PA counselling in resource-limited settings. A study supported that technology infrastructure, digital literacy, funding, human resources, organizational and management policies, and information technology security were considerable issues of eHealth technologies in developing countries. ${ }^{59}$

The findings of this qualitative study relied on the perspectives of physicians and nurses. However, resource- 
limited PHC teams may use a supportive approach, connecting with external providers (such as exercise physiologists or trainers, behavioral counsellors, and local community members) to reduce the health care burden. ${ }^{60}$ To optimize this process, an eHealth tool that can link PA counselling at PHC settings with external resources should be considered. These connections may enhance the continuity of care of patients with chronic illnesses.

\section{Recommendations}

Several eHealth tools were designed and implemented for PA counselling in PHC settings in high-income countries. ${ }^{52}$ Adoption of those eHealth tools has several considerations. Although some barriers are common among different PHC settings, the challenges are diverse. For example, a limitation of time is one of the most common barriers to PA counselling. A consultation provided by primary care physicians is very brief in low- and middle-income countries (48 seconds in Bangladesh) compared with a longer consultation in high-income countries (22.5 minutes in Sweden). ${ }^{61}$ This reflects the different needs among PHC settings. Therefore, developing a new eHealth tool requires the understanding of providers' perspectives as well as the limitations of PHC settings.

In the context of PHC settings in Thailand, PA counselling is considered an essential intervention for patients. The needs to improve the process of PA counselling include training, tools, and teams. ${ }^{4}$ A newly developed eHealth should function as a tool to support services. An eHealth tool that embeds the evidence-based information in its process has the potential to facilitate PA counselling as a trained facilitator for patients and providers. In addition, an effective eHealth tool can be a substitution of staff in the resource-limited settings. ${ }^{14}$

An eHealth tool for PA counselling that can be embedded into an electronic medical record is a preferred product among PHC providers. The challenge is how to create a universal eHealth tool that can work with different electronic medical record systems in different settings. A mobile application is another potential form. An application can be utilized by both patients and providers. An eHealth application should be able to use on low-cost mobile devices. The contents of the application should be practical and easy to understand.

\section{Limitations}

The study had some limitations. First, the major limitation was a small number of participants and imbalance in the distribution by gender and by years of experience. Within the four NCD clinics in the resource-limited settings. Each NCD clinic consisted of three to five nurses. Physicians were rotated to operate the NCD clinics due to a shortage of staff. The authors attempted to invite as many physicians and nurses as possible to participate in this study. Within the largest community hospital, the authors invited both NCD clinic teams to enhance the number of participants. Second, each FGD consisted of a small number of participants (two to four). To enhance participant interaction and the depth of the data collected, the NCD nurses took part in FGDs because eHealth PA counselling technology was a new and unfamiliar topic to them, so IDIs were less likely to be suitable. Although at least three nurses were invited to each FGD (to try to ensure that they were representative of the NCD nursing staff, as each clinic comprises three to five nurses), one FGD involved only two participants because one nurse was unavailable due to an unplanned meeting. Third, although this study intended to obtain transferability, the qualitative approach may not allow the findings to be fully generalized to other PHC settings. Nevertheless, the findings may help to identify concerns about essential elements to consider when developing an eHealth tool for use in the resource-limited settings.

\section{Conclusion}

The findings of this study highlight the PHC providers' perspectives on the development of an eHealth tool for PA counselling in the resource-limited settings. To improve PA counselling practice, a well-designed eHealth tool has the potential to support the practice. An automated PA prescription function as well as tracking and recall functions are expected to see in a new eHealth tool. Ease of use is considered as an important element. A new eHealth tool is expected to diminish barriers to PA counselling. Future research should focus on the usability and utility as well as the process evaluation of the PA counselling eHealth tool that will be developed based on this research.

\section{Acknowledgments}

This study was funded by The Medical Association of Thailand. The authors wish to thank all participants for their participation, and Aekthawat Watthanachon for his help with the research administration. This research was financially supported by the new strategic research project (P2P), Walailak University, Thailand. 


\section{Funding}

This study was part of a research project funded by The Medical Association of Thailand. The funding body had no role in the design, execution, interpretation, or writing of the study.

\section{Disclosure}

Dr Apichai Wattanapisit reports grants from The Medical Association of Thailand and the new strategic research project (P2P), Walailak University. The authors report no other potential conflicts of interest in this work.

\section{References}

1. Searight HR. Counseling patients in primary care: evidence-based strategies. Am Fam Physician. 2018;98:719-728.

2. Bowman MA, Neale AV. Family physicians are complex care physicians and quality of care advancement experts. J Am Board Fam Med. 2014;27(1):1-3. doi:10.3122/jabfm.2014.01.130283

3. Nabalamba A, Millar WJ. Going to the doctor. Health Rep. 2007; 18:23-35.

4. Wattanapisit A, Thanamee S, Wongsiri S. Physical activity counselling among GPs: a qualitative study from Thailand. BMC Fam Pract. 2019;20(1):72. doi:10.1186/s12875-019-0968-x

5. Lion A, Vuillemin A, Thornton JS, et al. Physical activity promotion in primary care: a Utopian quest? Health Promot Int. 2019;34 (4):877-886. doi:10.1093/heapro/day038

6. Hébert ET, Caughy MO, Shuval K. Primary care providers' perceptions of physical activity counselling in a clinical setting: a systematic review. Br J Sports Med. 2012;46:625-631. doi:10.1136/bjsports-2011-090734

7. Shuval K, Leonard T, Drope J, et al. Physical activity counseling in primary care: insights from public health and behavioral economics. CA Cancer J Clin. 2017;67(3):233-244. doi:10.3322/caac.21394

8. Writing Group for the Activity Counseling Trial Research Group. Effects of physical activity counseling in primary care: the activity counseling trial: a randomized controlled trial. JAMA. 2001;286 (6):677-687. doi:10.1001/jama.286.6.677

9. Wattanapisit A, Wattanapisit S, Wongsiri S. Overview of physical activity counseling in primary care. Korean J Fam Med. 2020 doi: $10.4082 / \mathrm{kjfm} .19 .0113$

10. Omura JD, Bellissimo MP, Watson KB, Loustalot F, Fulton JE, Carlson SA. Primary care providers' physical activity counseling and referral practices and barriers for cardiovascular disease prevention. Prev Med. 2018;108:115-122. doi:10.1016/j.ypmed.2017. 12.030

11. Young AJ. New technologies and general practice. Br J Gen Pract. 2016;66(653):601-602. doi:10.3399/bjgp16X688021

12. World Health Organization. WHO Guideline: Recommendations on Digital Interventions for Health System Strengthening. Geneva, Switzerland: World Health Organization; 2019.

13. van der Kleij RMJJ, Kasteleyn MJ, Meijer E, et al. SERIES: eHealth in primary care. Part 1: concepts, conditions and challenges. Eur J Gen Pract. 2019;25:179-189. doi:10.1080/13814788.2019.1658190

14. Wattanapisit A, Teo CH, Wattanapisit S, et al. Can mobile health apps replace GPs? A scoping review of comparisons between mobile apps and GP tasks. BMC Med Inform Decis Mak. 2020;20(1):5. doi:10.1186/s12911-019-1016-4

15. Prochaska JJ, Zabinski MF, Calfas KJ, et al. PACE+: interactive communication technology for behavior change in clinical settings. Am J Prev Med. 2000;19:127-131. doi:10.1016/S0749-3797(00)00 187-2
16. Calfas KJ, Sallis JF, Zabinski MF, et al. Preliminary evaluation of a multicomponent program for nutrition and physical activity change in primary care: PACE + for adults. Prev Med. 2002;34:153-161. doi:10.1006/pmed.2001.0964

17. Pinto BM, Friedman R, Marcus BH, et al. Effects of a computer-based, telephone-counseling system on physical activity. Am J Prev Med. 2002;23(2):113-120. doi:10.1016/S0749-3797(02) 00441-5

18. Carlfjord S, Nilsen P, Leijon M, et al. Computerized lifestyle intervention in routine primary health care: evaluation of usage on provider and responder levels. Patient Educ Couns. 2009;75(2):238-243. doi:10.1016/j.pec.2008.10.004

19. Glynn LG, Hayes PS, Casey M, et al. Effectiveness of a smartphone application to promote physical activity in primary care: the SMART MOVE randomised controlled trial. Br J Gen Pract. 2014;64(624): e384-e391. doi:10.3399/bjgp14X680461

20. van der Weegen S, Verwey R, Spreeuwenberg M, et al. It's LiFe! Mobile and web-based monitoring and feedback tool embedded in primary care increases physical activity: a cluster randomized controlled trial. J Med Internet Res. 2015;17(7):e184. doi:10.2196/ jmir.4579

21. Verwey R, van der Weegen S, Spreeuwenberg M, et al. Process evaluation of physical activity counselling with and without the use of mobile technology: a mixed methods study. Int J Nurs Stud. 2016;53:3-16. doi:10.1016/j.ijnurstu.2015.10.008

22. Gill DP, Blunt W, Silva N, et al. The HealtheSteps ${ }^{\mathrm{TM}}$ lifestyle prescription program to improve physical activity and modifiable risk factors for chronic disease: a pragmatic randomized controlled trial. BMC Public Health. 2019;19(1):841. doi:10.1186/s12889-019-7141-2

23. Anhoj J, Jensen AH. Using the internet for life style changes in diet and physical activity: a feasibility study. J Med Internet Res. 2004;6 (3):e28. doi:10.2196/jmir.6.3.e28

24. Parekh S, Vandelanotte C, King D, et al. Improving diet, physical activity and other lifestyle behaviours using computer-tailored advice in general practice: a randomised controlled trial. Int J Behav Nutr Phys Act. 2012;9(1):108. doi:10.1186/1479-5868-9-108

25. Parekh S, King D, Boyle FM, et al. Randomized controlled trial of a computer-tailored multiple health behaviour intervention in general practice: 12-month follow-up results. Int J Behav Nutr Phys Act. 2014;11(1):41. doi:10.1186/1479-5868-11-41

26. Wattanapisit A, Saengow U. Patients' perspectives regarding hospital visits in the universal health coverage system of Thailand: a qualitative study. Asia Pac Fam Med. 2018;17:9. doi:10.1186/ s12930-018-0046-X

27. Bradshaw C, Atkinson S, Doody O. Employing a qualitative description approach in health care research. Glob Qual Nurs Res. 2017;4:2333393617742282. doi:10.1177/2333393617742282

28. Matua GA, Van Der Wal DM. Differentiating between descriptive and interpretive phenomenological research approaches. Nurse Res. 2015;22:22-27. doi:10.7748/nr.22.6.22.e1344

29. O'Brien BC, Harris IB, Beckman TJ, et al. Standards for reporting qualitative research: a synthesis of recommendations. Acad Med. 2014;89(9):1245-1251. doi:10.1097/ACM.0000000000000388

30. Tong A, Sainsbury P, Craig J. Consolidated criteria for reporting qualitative research (COREQ): a 32-item checklist for interviews and focus groups. Int J Qual Health Care. 2007;19(6):349-357. doi:10.1093/intqhe/mzm042

31. Saunders B, Sim J, Kingstone T, et al. Saturation in qualitative research: exploring its conceptualization and operationalization. Qual Quant. 2018;52:1893-1907. doi:10.1007/s11135-017-0574-8

32. Braun V, Clarke V. Using thematic analysis in psychology. Qual Res Psychol. 2006;3:77-101. doi:10.1191/1478088706qp063oa

33. Gale NK, Heath G, Cameron E, et al. Using the framework method for the analysis of qualitative data in multi-disciplinary health research. BMC Med Res Methodol. 2013;13(1):117. doi:10.1186/ 1471-2288-13-117 
34. Casey M, Hayes PS, Glynn F, et al. Patients' experiences of using a smartphone application to increase physical activity: the SMART MOVE qualitative study in primary care. Br J Gen Pract. 2014;64 (625):e500-e508. doi:10.3399/bjgp14X680989

35. Choo S, Kim JY, Jung SY, et al. Development of a weight loss mobile app linked with an accelerometer for use in the clinic: usability, acceptability, and early testing of its impact on the patient-doctor relationship. JMIR Mhealth Uhealth. 2016;4:e24. doi:10.2196/ mhealth. 4546

36. Garcia-Ortiz L, Recio-Rodriguez JI, Agudo-Conde C, et al. Longterm effectiveness of a smartphone app for improving healthy lifestyles in general population in primary care: randomized controlled trial (Evident II Study). JMIR Mhealth Uhealth. 2018;6:e107. doi:10.2196/mhealth.9218

37. Glynn LG, Glynn F, Casey M, et al. Implementation of the SMART MOVE intervention in primary care: a qualitative study using normalisation process theory. BMC Fam Pract. 2018;19:48. doi:10.1186/ s12875-018-0737-2

38. Recio-Rodriguez JI, Agudo-Conde C, Martin-Cantera C, et al. Shortterm effectiveness of a mobile phone app for increasing physical activity and adherence to the mediterranean diet in primary care: a randomized controlled trial (EVIDENT II Study). J Med Internet Res. 2016;18(12):e331. doi:10.2196/jmir.6814

39. Verwey R, van der Weegen S, Spreeuwenberg M, et al. Technology combined with a counseling protocol to stimulate physical activity of chronically ill patients in primary care. Stud Health Technol Inform. 2014;201:264-270.

40. Becker A, Herzberg D, Marsden N, et al. A new computer-based counselling system for the promotion of physical activity in patients with chronic diseases-results from a pilot study. Patient Educ Couns. 2011;83(2):195-202. doi:10.1016/j.pec.2010.05.024

41. Carroll JK, Lewis BA, Marcus BH, et al. Computerized tailored physical activity reports. a randomized controlled trial. Am J Prev Med. 2010;39(2):148-156. doi:10.1016/j.amepre.2010.04.005

42. De Cocker K, Spittaels H, Cardon G, et al. Web-based, computer-tailored, pedometer-based physical activity advice: development, dissemination through general practice, acceptability, and preliminary efficacy in a randomized controlled trial. $J$ Med Internet Res. 2012;14(2):e53. doi:10.2196/jmir.1959

43. Abu-Saad K, Murad H, Barid R, et al. Development and efficacy of an electronic, culturally adapted lifestyle counseling tool for improving diabetes-related dietary knowledge: randomized controlled trial among ethnic minority adults with type 2 diabetes mellitus. $J$ Med Internet Res. 2019;21:e13674. doi:10.2196/13674

44. Carlfjord S, Johansson K, Bendtsen P, et al. Staff perspectives on the use of a computer-based concept for lifestyle intervention implemented in primary health care. Health Educ J. 2010;69(3):246-256. doi:10.1177/0017896910364883

45. Christian JG, Byers TE, Christian KK, et al. A computer support program that helps clinicians provide patients with metabolic syndrome tailored counseling to promote weight loss. J Am Diet Assoc. 2011;111:75-83. doi:10.1016/j.jada.2010.10.006

46. Degroote L, Plaete J, De Bourdeaudhuij I, et al. The effect of the eHealth intervention 'MyPlan 1.0' on physical activity in adults who visit general practice: a quasi-experimental trial. Int $J$ Environ Res Public Health. 2018;15:228. doi:10.3390/ijerph15020228
47. Diaz VA, Mainous AG 3rd, Gavin JK, et al. Use of a tablet-based risk assessment program to improve health counseling and patient-provider relationships in a federally qualified health center. Am J Med Qual. 2016;31(5):434-440. doi:10.1177/10628606155 87012

48. Leijon M, Arvidsson D, Nilsen P, et al. Improvement of physical activity by a kiosk-based electronic screening and brief intervention in routine primary health care: patient-initiated versus staff-referred. J Med Internet Res. 2011;13(4):e99. doi:10.2196/jmir.1745

49. Mann DM, Palmisano J, Lin JJ. A pilot randomized trial of technology-assisted goal setting to improve physical activity among primary care patients with prediabetes. Prev Med Rep. 2016;4:107-112. doi:10.1016/j.pmedr.2016.05.012

50. Poppe L, Plaete J, Huys N, et al. Process evaluation of an eHealth intervention implemented into general practice: general practitioners' and patients' views. Int J Environ Res Public Health. 2018;15 (7):1475. doi:10.3390/ijerph15071475

51. Sciamanna CN, Marcus BH, Goldstein MG, et al. Feasibility of incorporating computer-tailored health behaviour communications in primary care settings. Inform Prim Care. 2004;12:40-48. doi:10.14236/jhi.v12i1.107

52. Wattanapisit A, Tuangratananon T, Wattanapisit S. Usability and utility of eHealth for physical activity counselling in primary health care: a scoping review. BMC Fam Pract. 2020;21:229. doi:10.1186/ s12875-020-01304-9

53. Boers SN, Jongsma KR, Lucivero F, et al. SERIES: eHealth in primary care. Part 2: exploring the ethical implications of its application in primary care practice. Eur J Gen Pract. 2020;26:26-32. doi: $10.1080 / 13814788.2019 .1678958$

54. Kleinpeter E. Four ethical issues of "E-Health". IRBM. 2017;38:245-249. doi:10.1016/j.irbm.2017.07.006

55. Shaw T, McGregor D, Brunner M, et al. What is eHealth (6)? Development of a conceptual model for eHealth: qualitative study with key informants. J Med Internet Res. 2017;19(10):e324. doi:10.2196/jmir.8106

56. Safi S, Thiessen T, Schmailzl KJG. Acceptance and resistance of new digital technologies in medicine: qualitative study. JMIR Res Protoc. 2018;7(12):e11072. doi:10.2196/11072

57. de Grood C, Raissi A, Kwon Y, et al. Adoption of e-health technology by physicians: a scoping review. J Multidiscip Healthc. 2016;9:335-344. doi:10.2147/JMDH.S103881

58. Granja C, Janssen W, Johansen MA. Factors determining the success and failure of eHealth interventions: systematic review of the literature. J Med Internet Res. 2018;20:e10235. doi:10.2196/10235

59. Zayyad MA, Toycan M. Factors affecting sustainable adoption of e-health technology in developing countries: an exploratory survey of Nigerian hospitals from the perspective of healthcare professionals. PeerJ. 2018;6:e4436. doi:10.7717/peerj.4436

60. AuYoung M, Linke SE, Pagoto S, et al. Integrating physical activity in primary care practice. Am J Med. 2016;129(10):1022-1029. doi:10.1016/j.amjmed.2016.02.008

61. Irving G, Neves AL, Dambha-Miller H, et al. International variations in primary care physician consultation time: a systematic review of 67 countries. BMJ Open. 2017;7(10):e017902. doi:10.1136/bmjopen2017-017902 


\section{Publish your work in this journal}

The Journal of Multidisciplinary Healthcare is an international, peerreviewed open-access journal that aims to represent and publish research in healthcare areas delivered by practitioners of different disciplines. This includes studies and reviews conducted by multidisciplinary teams as well as research which evaluates the results or conduct of such teams or healthcare processes in general. The journal

covers a very wide range of areas and welcomes submissions from practitioners at all levels, from all over the world. The manuscript management system is completely online and includes a very quick and fair peer-review system. Visit http://www.dovepress.com/testimonials. php to read real quotes from published authors. 